\title{
Fabrication of Nano-Tips by Carbon Contamination in a Scanning Electron Microscope for Use in Scanning Probe Microscopy and Field Emission
}

\author{
Massimo Antognozzi, Andrea Sentimenti and Ugo Valdrè \\ INFM and Centre of Electron Microscopy, Department of Physics of the University, \\ via Irnerio 46, 40126 Bologna, Italy
}

(Received August 29, 1997; accepted December 1, 1997)

PACS.61.16.-d - Electron, ion, and scanning probe microscopy

PACS.79.70.+q - Field emission, ionization, evaporation, and desorption

\begin{abstract}
Results are reported on a systematic study addressed to an effective fabrication of nano-tips by means of carbon contamination in a scanning electron microscope. Nano-tips with angular aperture of $10^{\circ}$, apical radius of about $5 \mathrm{~nm}, 1 \mu \mathrm{m}$ long can be efficiently produced by our method in less than $60 \mathrm{~s}$ of electron beam exposure; it involves, in particular, successive focusing during tip growth and the use of a carbon block as a source of contaminant. These tips have been used as high aspect ratio and low capillary force probes in atomic force microscopy, and as nano-sized field emitters for electron guns.
\end{abstract}

\section{Introduction}

There is nowadays a wide interest in the fabrication of nano-sized tips. At least three major areas are concerned with their applications: (i) Scanning Probe Microscopy (SPM) [1], (ii) field emission [2,3] and (iii) field emitter displays [4]. Here we deal specifically with the first two cases only; however case (iii) will benefit from progress in field (ii).

Case (i) requires probes with the highest possible aspect ratio (i.e., the smallest tip angular aperture), mechanical strength and the smallest apical radius in order to profile irregular surfaces with steep slants and to attain high resolution. An additional requirement may be the electrical conductivity when the probes are to be used in scanning tunneling microscopy and electric force microscopy.

Case (ii) demands sharp and electrically conducting emitters, in order to get the highest possible current densities and coherence, with the lowest extraction voltage, for use in electron guns applied in various types of microscopy and devices.

Usually, probes are made out of metals (e.g., W, Pt, Pt/Ir), semiconductors (Si) or insulators $\left(\mathrm{Si}_{3} \mathrm{~N}_{4}\right)$; the latters are sometimes coated with a conducting layer (e.g., $\mathrm{Au}, \mathrm{Pd}, \mathrm{Al}$ ) or a magnetic layer $(\mathrm{Ni}, \mathrm{Co})$ depending on the application. Emitters are made of metals or semiconductors. Here we deal with both probe and emitter tips, fabricated by carbon contamination in a Scanning Electron Microscope (SEM). Whereas this method has been pursued in several places as far as probes are concerned, nothing has been reported to our knowledge 
in the case of field emitters. However, the properties of field emitters made of carbon in the form of nanotubes and diamond chips have been investigated, and the results obtained are very interesting [4-6]. This paper reports first on the deliberate enhancement of carbon contamination in an SEM, then on an optimized method for the fabrication of carbon nano-tips; examples of their applications as probes and field emitters are finally presented.

\section{Carbon Contamination}

Carbon contamination has been studied extensively in electron and ion microscopy with the aim to reduce and ideally eliminate it. Very little work has been done so far on the opposite problem of increasing the contamination selectively, efficiently and in a simple way; such previous work concerns the production of high aspect ratio probes at the top of cantilevers for application in scanning probe microscopy by using the electron beam of a SEM. The inconsistent results we obtained by following empirical recipes suggested by various authors (i.e., use of several contaminants, such as paraffin oils or blocks), prompted us to tackle the problem afresh.

The following parameters affecting the growth of carbon contamination tips were taken into account and their effects systematically investigated.

1. SEM accelerating voltage,

2. beam current, measured with a Faraday cup,

3. beam diameter, at specimen eucentric level, and corresponding current density,

4. beam stability, focus and astigmatism,

5. specimen material, shape and clamping,

6. stub material,

7. coating before carbon-growth for non-conductive specimens.

The pressure in the specimen chamber during the investigations ranged from $5 \times 10^{-5}$ to $1 \times 10^{-4}$ Torr and the hydrocarbon partial pressure was about $10^{-6}$ Torr, measured with a mass spectrometer [7].

The assessment of the tips geometry after their growth was usually made by SEM observations with the tip placed at $90^{\circ}$ with respect to the beam; in some cases the observations were performed in a Transmission Electron Microscope (TEM).

The results are reported below.

(i) The best accelerating voltage is $30 \mathrm{kV}$ (highest value provided by our instrument, a Philips SEM 515); the beam current at the specimen level ranged from about $10^{-12}$ to $10^{-13} \mathrm{~A}$ and the corresponding nominal spot sizes were $20 \mathrm{~nm}$ and $5 \mathrm{~nm}$ (the emission current, read from the microscope meter, was between 100 and $200 \mu \mathrm{A}$ ).

Since it is well known that the contamination rate $C$ increases on decreasing the accelerating voltage $V\left(C\right.$ is proportional to $V^{-0.8}$, Fig. 1a) $[8,9]$ and is proportional to the current density $J$ at specimen level, one would expect the best results are obtained at low voltages. However, the gain in contamination at low voltage does not balance the contamination reduction due to the decrease of current density connected with the voltage change (Fig. 1b) [8,9]. In fact, the contamination decreases by a factor of about 2.5 by lowering the voltage from 30 to $15 \mathrm{kV}$.

Tips grown at the highest voltage show better features than those produced at low voltages, for the same values of the remaining free parameters: they have, of course, a greater height 

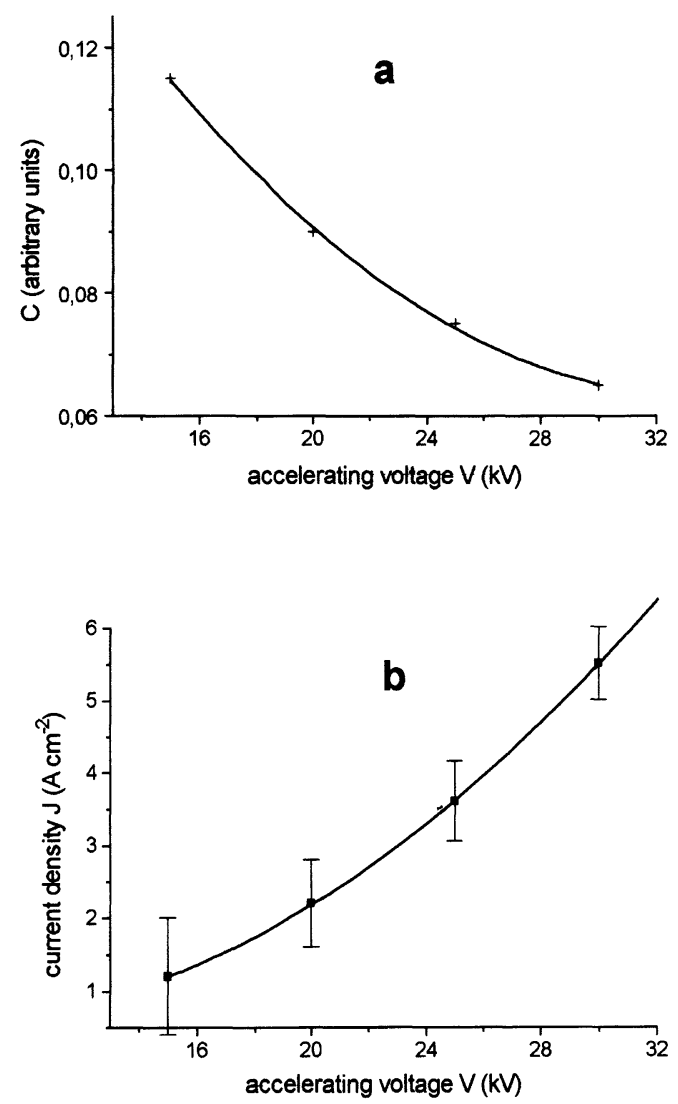

Fig. 1. - (a) Carbon contamination $C$ (arbitrary units) vs. accelerating voltage $V$. The trend follows the law $C \propto V^{-0.8}$. (b) Current density $J$ vs. accelerating voltage $V$. The experimental points have been interpolated by the curve $J=0.01 V^{2}-0.15 V+1.1$. Comparison between (a) and (b) shows that by increasing $V$ by a factor of 2 (from 15 to $30 \mathrm{kV}$ ) the contamination decreases by about $57 \%$, whereas the current density increases by a factor of 5 , with a net gain in contamination by a factor of 2.5 .

and, in addition, a smaller cross-section at their base and a smaller apical angle, probably due to the smaller flanks of the beam cross-section than in the case of low voltage beams. An example is reported in Figure 2. It is anticipated that the use of accelerating voltages above $30 \mathrm{kV}$ should be advantageous.

(ii) The smallest spot size must be used (Fig. 3).

(iii) The beam has to be well focused on the sample and well stabilised (both in position and in emission); this is obtained by running it for about 1 hour before growing the tips. The goniometer stage must be clamped, the traverse stage stable and the astigmatism carefully corrected (Fig. 4). The astigmatism correction is made at a magnification of $20.000 \div 40.000$ $\times$ by observing latex spheres of $1 \mu$ m diameter, coated with a thin layer of gold $(12 \div 20 \mathrm{~nm})$. The round shape of the spheres helps in the correction. If instabilities are still present and the beam current is low, cleaning of the apertures and of the wehnelt usually improves the situation. These conditions are of paramount importance: specimen and beam drift hinder the formation of a good contamination cone. 


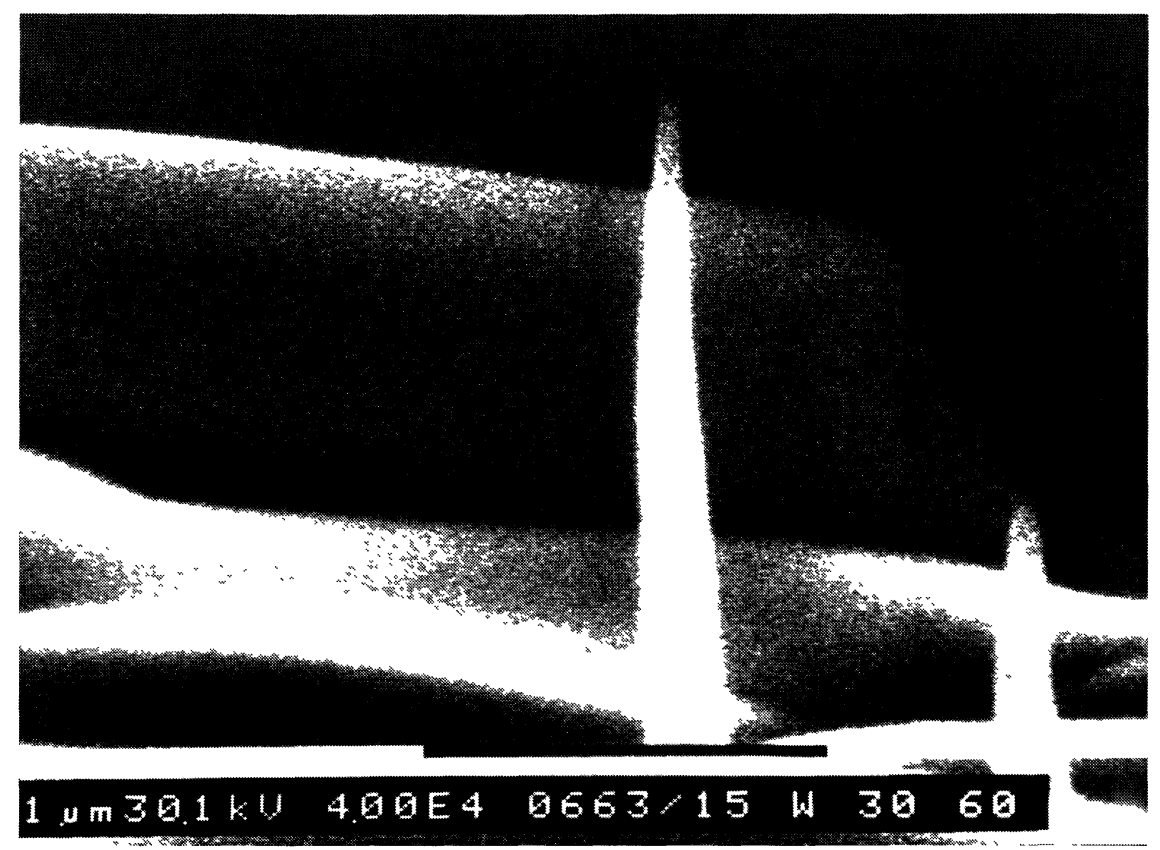

Fig. 2. - SEM secondary electron image taken at $30 \mathrm{kV}$ of two carbon tips grown on a tungsten surface showing a large difference of geometry and size. (Right side tip) Growth conditions: $15 \mathrm{kV}$, $60 \mathrm{~s}$, spot size $5 \mathrm{~nm}$, current density $1.1 \mathrm{~A} \mathrm{~cm}^{-2}$. (Left side tip) Growth conditions: $30 \mathrm{kV}, 60 \mathrm{~s}$, spot size $5 \mathrm{~nm}$, current density $5.6 \mathrm{~A} \mathrm{~cm}^{-2}$.

(iv) The specimen material and shape do not seem to influence the contamination growth, at least for the types which were tested (Tungsten in the form of wires, plates and microscope filaments, Tantalum edges, Gold and Palladium films) as long as the specimen is an electrical conductor and clean. In the case of $\mathrm{Si}$ and $\mathrm{Si}_{3} \dot{\mathrm{N}}_{4}$ cantilevers, effective C-contamination growth is obtained by first coating the substrate with a metal layer (about $20 \mathrm{~nm}$ thick of $\mathrm{Au}$ or $\mathrm{Pd})$ to avoid charging up. The carbon growth is more regular on a $\mathrm{Pd}$ than on a $\mathrm{Au}$ coating film, owing to the finest size of the Pd clusters. If the above specified conditions are not well set, contamination rate is low, even with the microscope in standard order; after 10 min of irradiation only thin $(20 \div 80 \mathrm{~nm})$ slightly bulging contamination spots of $50 \div 80 \mathrm{~nm}$ diameter were obtained.

(v) Whether the specimen is mechanically clamped or glued with silver dag is irrelevant. On the contrary, the use of a carbon stub (with the specimen mechanically held), improves the contamination growth and the reproducibility of the results. Alternatively, simply and cheaply, use can be made of standard $\mathrm{Al}$ stubs to support the specimen and by placing in its vicinity a small carbon block $\left(\sim 50 \mathrm{~mm}^{3}\right)$. The effectiveness of the C-block depends on both its surface and its distance from the specimen region where the $\mathrm{C}$-growth has to take place. The growth rate is $1 \div 2 \mu \mathrm{m}$ per minute. The adsorption properties of carbon have been known and exploited for long time. In the present situation it is believed that under vacuum the carbon block releases the contaminants previously adsorbed, thus creating a high concentration of carbon compounds in the proximity of the specimen, which favours the contamination growth [10]. The contamination effect is also well noticeable when a large C-block (surface $50 \mathrm{~cm}^{2}$ ) is placed inside the specimen chamber (e.g., in a corner). 


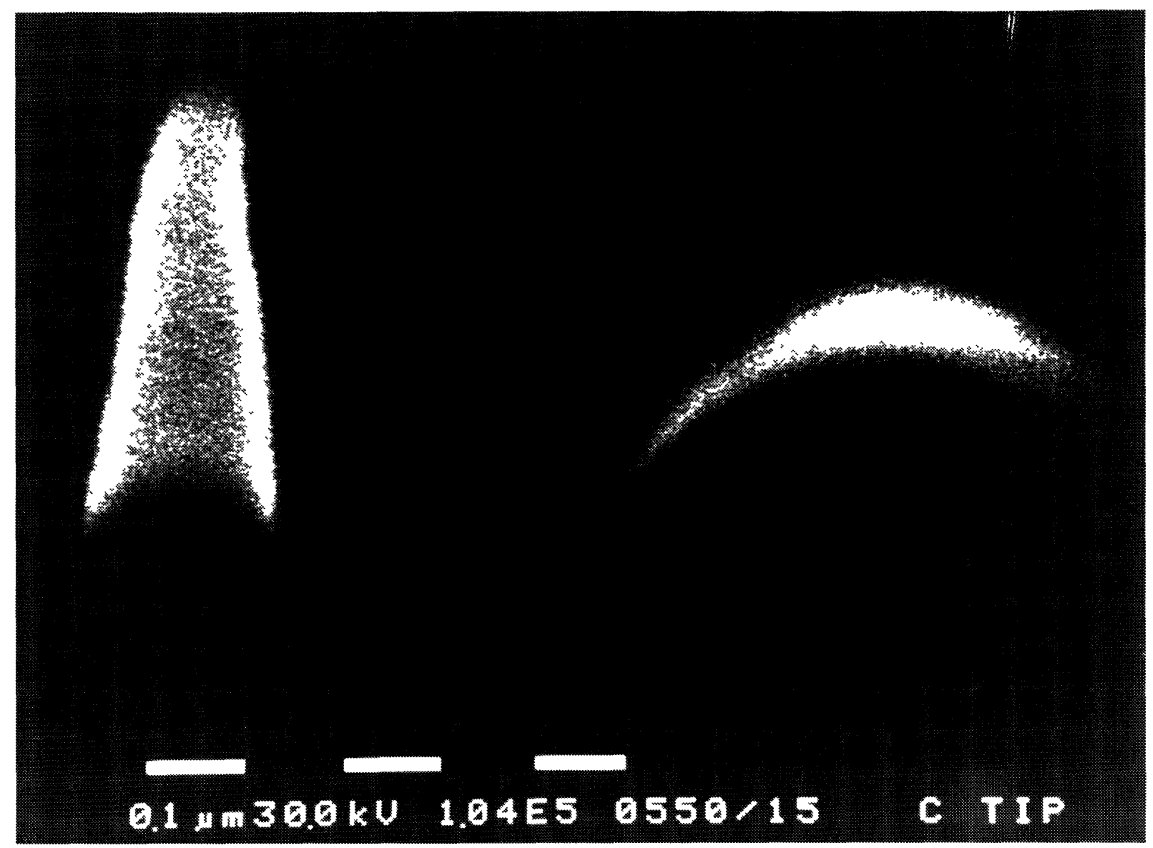

Fig. 3. - SEM secondary electron image of a tip grown with a $5 \mathrm{~nm}$ beam diameter (left), and of the contamination spot (right) which was produced during an attempt to grow a carbon tip with a large electron beam diameter $(100 \mathrm{~nm})$.

(vi) Charging up effects constitute a serious problem when using high accelerating voltages, since they affect the stability of the electron beam and give rise to drift. The coating of the substrate with a metal (e.g., gold) overcomes this problem. We also found that coating obtained by evaporation provides more reproducible results than sputtering.

\section{Carbon-Tip Growth}

The practical procedure developed to fabricate conically shaped C-tip with small full aperture angle $\left(<10^{\circ}\right)$ and a small apical radius $(<5 \mathrm{~nm})$ is as follows.

First of all the electron beam, set at the smallest spot, is checked to be spatially and intensity stable (time required usually $1 \mathrm{~h}$ ) and the astigmatism corrected with the help of latex spheres. The specimen on which the C-tips have to be grown is then inserted in the SEM. The electron beam is focused over an area where small details are present (observation made at $80.000 \div 160.000 \times)$ near to the region where the tip has to be grown. Then the beam is stopped on the latter region. As soon as contamination is formed, astigmatism correction is again performed by observing the contamination spot. Good focusing, high stability and careful astigmatism correction are essential operations in order to achieve high quality tips; otherwise the regular shape, the sharpness and even the growth rate are jeopardised (Fig. 4).

During the growth of contamination, the Secondary Electron (SE) signal (observed in the $y$-monitor) increases because of topographic (edge) effects [11]; it increases until the tip has reached its maximum aspect ratio. The beam is then scanned and the geometrical quality of the tip assessed visually by tilting the specimen stage by $90^{\circ}$ with respect to the electron beam.

The finest results are, however, obtained by a focusing sequence: the beam is first scanned for selecting the region of growth and for focusing, then scanning is stopped and C-growth 


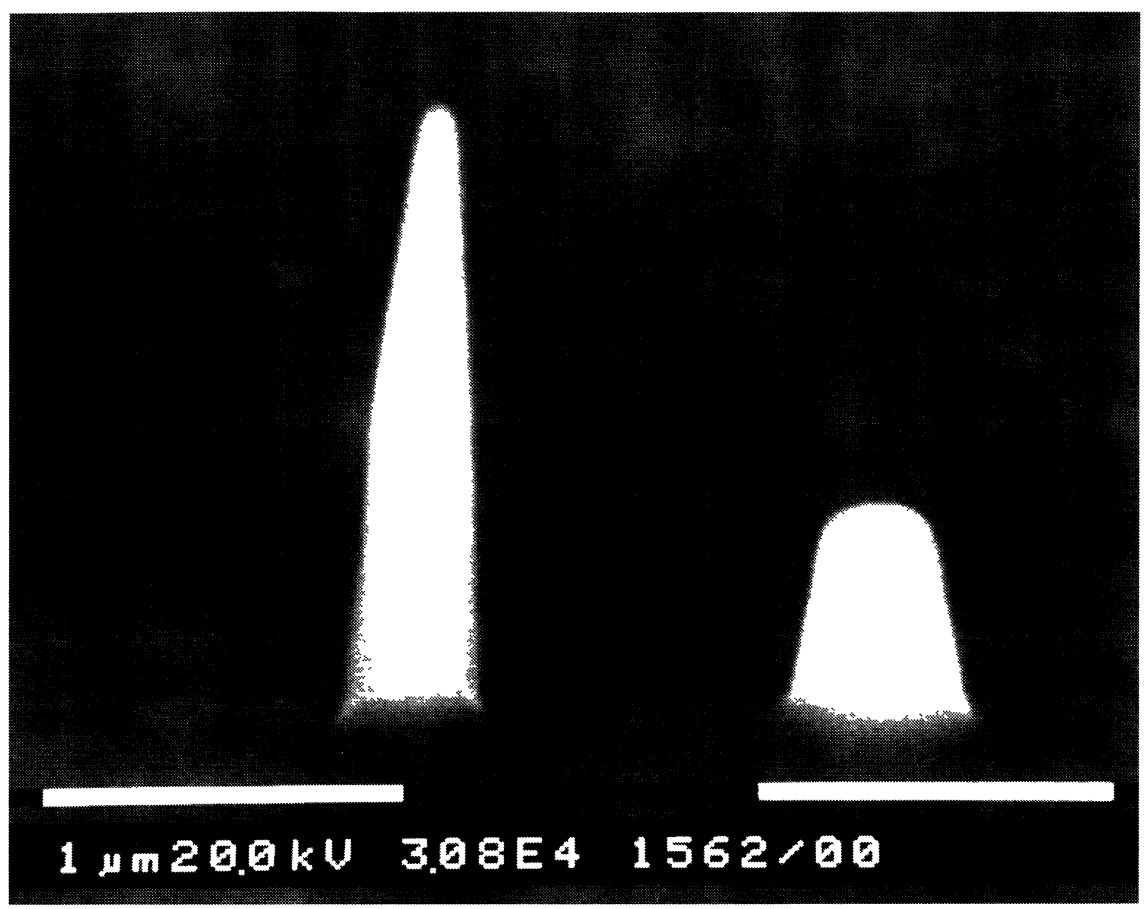

Fig. 4. - SEM secondary electron images of two C-tips grown under the same contamination conditions $(60 \mathrm{~s}, 20 \mathrm{kV})$ except that: (right) with good astigmatism correction and good focusing; (left) after slightly altering the astigmatism correction.

initiated for a short time $(\approx 20 \mathrm{~s})$; after that, scanning is resumed (thus the contamination process is drastically reduced), the magnification is increased for refocusing and then the Cgrowth is re-started by stopping the scanning again, and so on for 2 or three times. In other words the top of the initial C-cone is used as the starting point for the following contamination instalment (Fig. 5).

The results of the application of the two methods (just one focusing and several focusing procedures) show two different types of tip geometry. In the first case, the tip shape is that of a paraboloid rotationally symmetric; in the second case, the tip shape is that of a superposition of frustocones which end with a paraboloid (Fig. 5). Details of the tips fabricated with single and multi-focalisations have been obtained from high resolution images $(\sim 0.3 \mathrm{~nm})$ and examples are presented in Table I (see also Fig. 6a).

A particular case of growth is shown in Figure $6 \mathrm{~b}$, where a protuberance of radius $\approx 1 \mathrm{~nm}$ has been formed at the end of the tip, probably due to a small shift at the very end of the growth. We intend to investigate this fact which may lead to the formation of tip ends smaller than the beam dimension.

Nano-diffraction and high resolution imaging have demonstrated that the tips are formed by amorphous carbon.

The tilting stage may also be used to grow tips with their axis inclined with respect to the substrate surface normal (up to $\pm 60^{\circ}$ ), as in the case of $\mathrm{Si}_{3} \mathrm{~N}_{4}$ cantilevers (Fig. 7). This possibility may be relevant in profiling problems (see Sect. 4.1). 


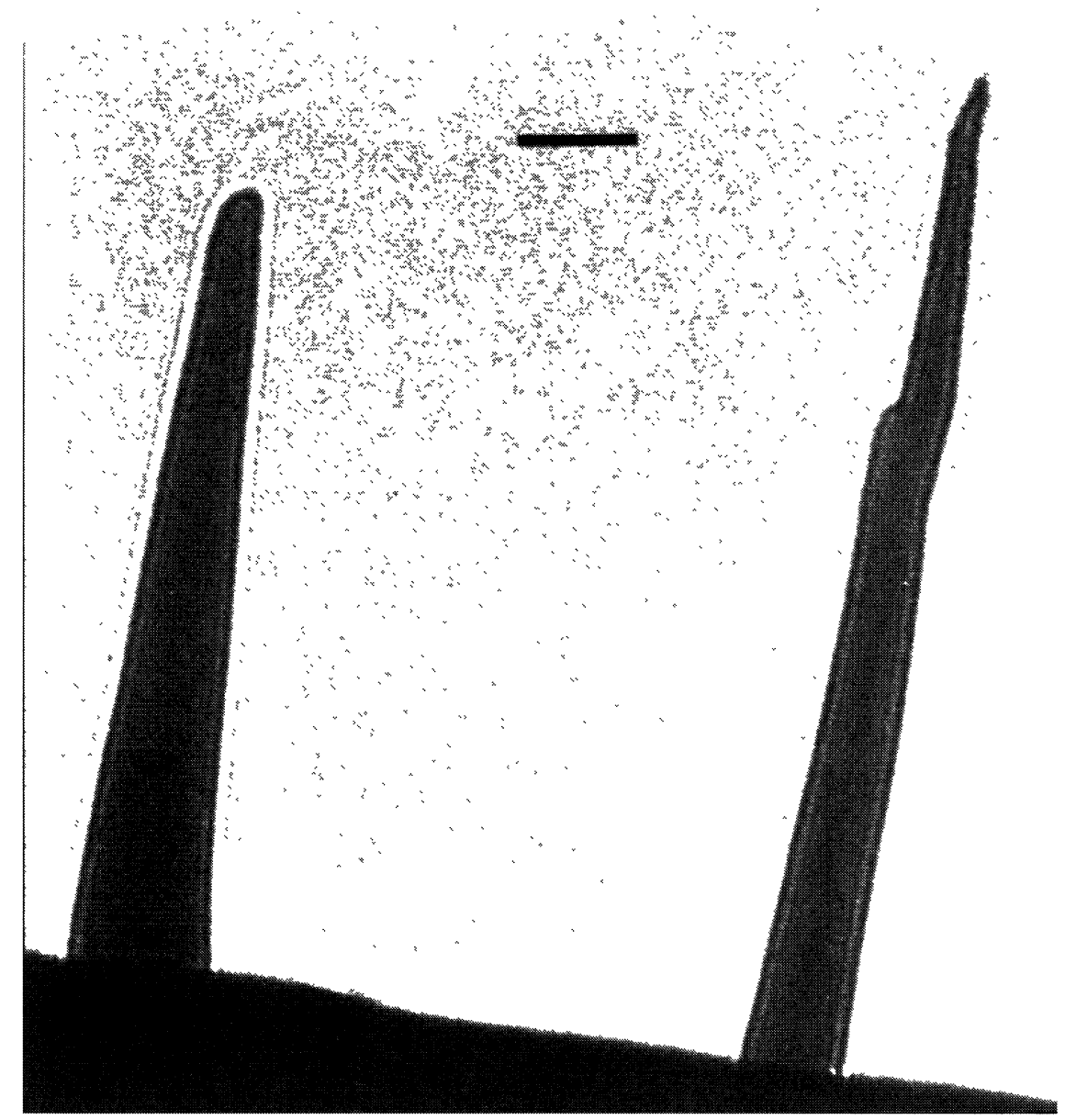

Fig. 5. - FEG/TEM images of two carbon tips grown in a SEM under the same conditions except for refocusing. (left) The regular shape of the tip (practically a frustocone ending with a hemisphere) indicates a single initial focalisation. (right) The irregular shape, formed by overlapping truncated cones, is the result of successive refocusing. The points where focusing has taken place correspond to the steps and changes in slope. Marker $=100 \mathrm{~nm}$.

Table I. - Geometrical carbon-tip parameters according to the focusing method used during growth.

\begin{tabular}{|c|c|c|c|c|c|c|}
\hline $\begin{array}{c}\text { Type } \\
\text { of } \\
\text { growth }\end{array}$ & $\begin{array}{c}\text { Tip } \\
\text { height } \\
\mathrm{nm}\end{array}$ & $\begin{array}{c}\text { Tip base } \\
\text { diameter } \\
\mathrm{nm}\end{array}$ & $\begin{array}{c}\text { Width at } \\
\text { half height } \\
\mathrm{nm}\end{array}$ & $\begin{array}{c}\text { Full } \\
\text { angle } \\
\text { degrees }\end{array}$ & $\begin{array}{c}\text { Apical } \\
\text { radius } \\
\mathrm{nm}\end{array}$ & $\begin{array}{c}\text { Growth } \\
\text { time } \\
\mathrm{s}\end{array}$ \\
\hline Single focalization & $500 \div 600$ & $100 \div 130$ & $60 \div 80$ & $8 \div 10$ & $10 \div 16$ & $20 \div 60$ \\
\hline Successive focalizations & $600 \div 1500$ & $50 \div 90$ & $30 \div 60$ & $4 \div 8$ & $2 \div 6$ & $20 \div 60$ \\
\hline
\end{tabular}




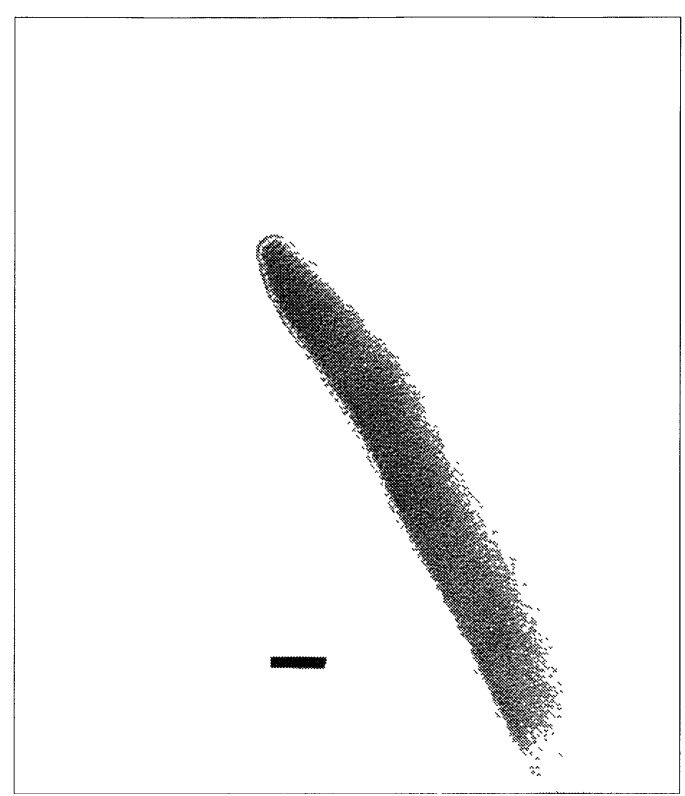

a)

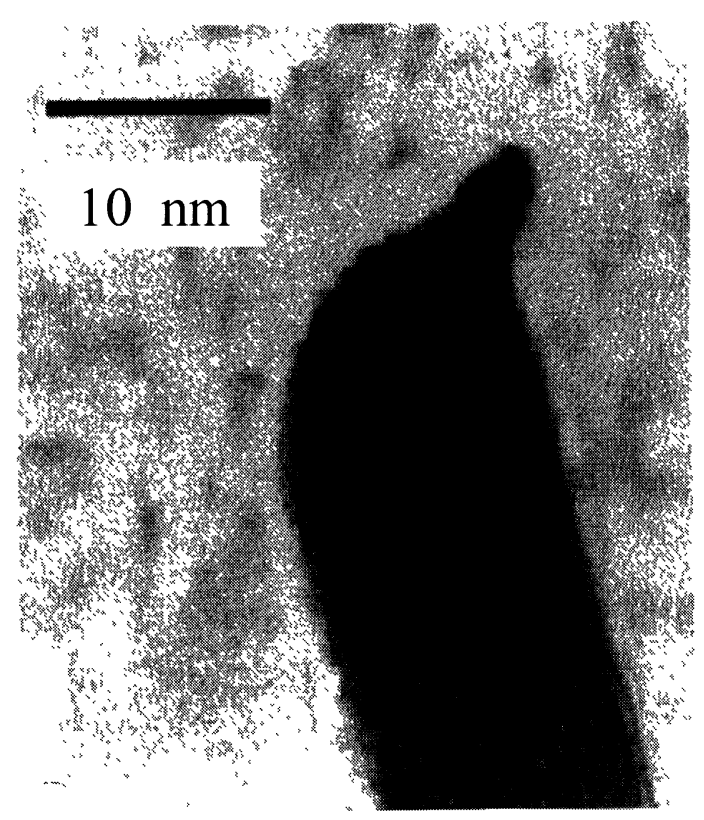

b)

Fig. 6. - (a) TEM/FEG high resolution image of a tip apex. The tip seems to be formed by amorphous carbon; this result has been confirmed by nanodiffraction. (b) TEM high resolution image of a tip apex ending with a small protuberance of radius about $1 \mathrm{~nm}$. The markers represent $10 \mathrm{~nm}$ in both images. 


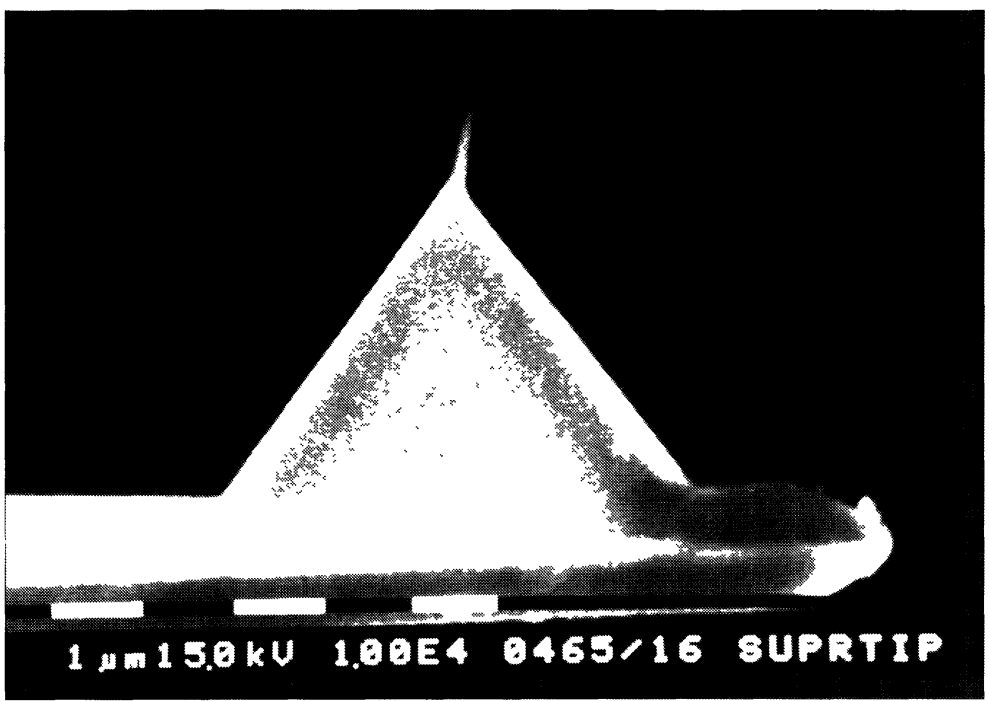

Fig. 7. - SEM secondary electron image of a nanoprobe fabricated by carbon contamination at the top of an $\mathrm{AFM} \mathrm{Si} \mathrm{N}_{4}$ pyramidal tip. Note that the nano-tip has been deliberately grown inclined by $10^{\circ}$ with respect to the pyramid axis, in order to perform scannings perpendicularly to the specimen surface.

\section{Applications}

Two applications are presented: (i) the case of topographic improvement and, (ii) the case of field emission.

\subsection{Topography}

The same specimen of known structure has been sequentially observed in an AFM with two different cantilever tips, but otherwise under the same experimental conditions. The specimen is a cross-grating of square pits (holes) having a size of $500 \pm 15 \mathrm{~nm}$ and a depth of $193.6 \pm 6.3 \mathrm{~nm}$ (as specified by the supplier); their spacing is $1 \mu \mathrm{m}$.

It was first observed in the contact mode by using conventional $\mathrm{Si}_{3} \mathrm{~N}_{4}$ type of cantilevers with a pyramidal tip (apex angle $68^{\circ}$ ) in Digital Instrument, Multimode III and then with similar types of cantilevers where at the top of the $\mathrm{Si}_{3} \mathrm{~N}_{4}$ pyramid a carbon nano-tip had been grown with the method described in Section 3 (Fig. 7). The growth angle was $10^{\circ}$ with respect to the pyramid axis, so that the scanning of the specimen was performed with the C-tip axis perpendicular to the sample surface.

From Figures $8 \mathrm{a}$ and $\mathrm{b}$, it is apparent the superior quality and accuracy of the image obtained with the carbon nano-tip, in particular with respect to the shape and size of the holes, and to the possibility of probing their bottoms. On the contrary, the images produced by the use of conventional tips clearly show the convolution of the hole with the tip shape: the (false) taper angle of the hole is the same as that of the pyramidal tip angle. Quantitative results worked out by observing a series of holes are reported in Tables II and III. Within the experimental and statistical errors, the dimensions of the holes are consistent with those supplied by the manufacturer of the grating, confirming that practically no artefacts have been produced by the nano-tips. The hole sizes reported in Table III have been measured at the top of the profile. 
Table II. - Hole depth.

\begin{tabular}{|c|c|c|}
\hline Hole & $\begin{array}{c}\text { Standard } \mathrm{Si}_{3} \mathrm{~N}_{4} \\
\text { pyramidal probe } \\
\text { Hole depth }(\mathrm{nm})\end{array}$ & C-nanotip \\
\hline 1 & 164 & 201 \\
2 & 165 & 199 \\
3 & 158 & 201 \\
4 & 157 & 200 \\
5 & 159 & 194 \\
6 & 160 & 197 \\
7 & 164 & 193 \\
8 & 168 & 194 \\
9 & 162 & 196 \\
10 & 162 & 193 \\
\hline Average depth and & $169 \pm 9$ & $197 \pm 9$ \\
statistical error $(3 \sigma)$ & \multicolumn{2}{|c|}{$193.6 \pm 6.3$} \\
\hline Manufacturer's data & \multicolumn{2}{|c|}{} \\
\hline
\end{tabular}

Table III. - Hole size.

\begin{tabular}{|c|c|c|}
\hline Hole & $\begin{array}{c}\text { Standard } \mathrm{Si}_{3} \mathrm{~N}_{4} \\
\text { pyramidal probe } \\
\text { Hole size }(\mathrm{nm})\end{array}$ & C-nanotip \\
\hline 1 & 429 & 503 \\
2 & 429 & 488 \\
3 & 424 & 490 \\
4 & 434 & 487 \\
5 & 427 & 479 \\
6 & 428 & 505 \\
7 & 433 & 488 \\
8 & 429 & 489 \\
9 & 435 & 488 \\
10 & 426 & 490 \\
\hline Average size and & $429 \pm 10$ & $491 \pm 22$ \\
statistical error $(3 \sigma)$ & \multicolumn{2}{|c|}{$500 \pm 15$} \\
\hline Manufacturer's data & \multicolumn{2}{|c|}{} \\
\hline
\end{tabular}


a
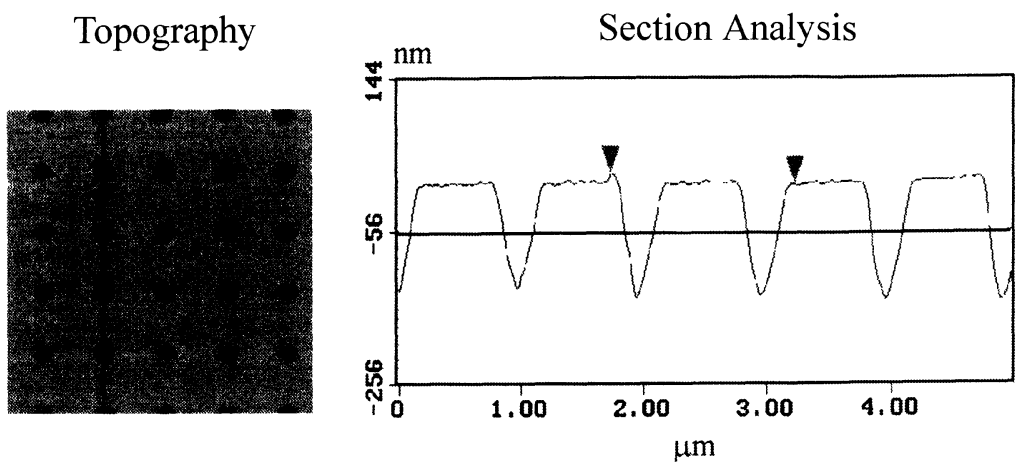

b
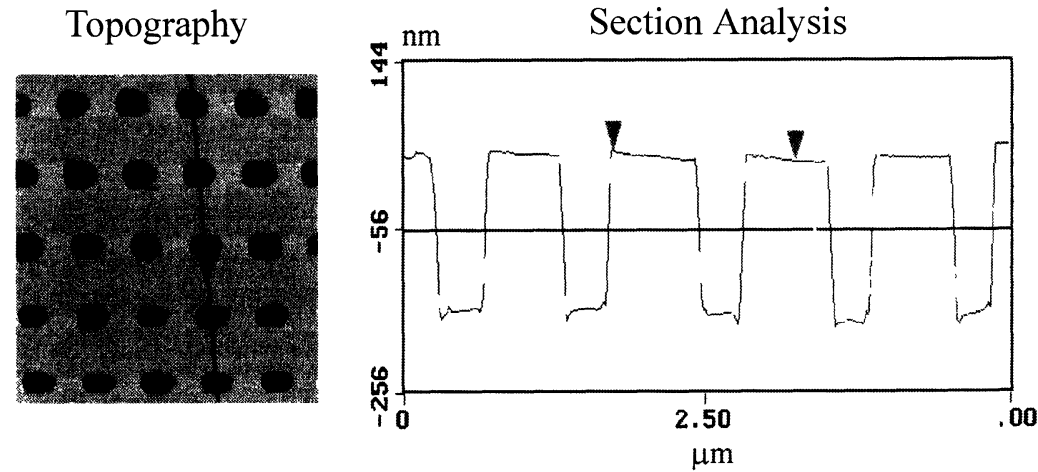

Fig. 8. - Cross-grating calibration standard with blind holes (pits). AFM topographic images acquired in the contact mode and corresponding sections ( $y$-modulated) along the vertical continuous line. (a) The probe is a standard $\mathrm{Si}_{3} \mathrm{~N}_{4}$ pyramidal tip. (b) The probe is a carbon nano-tip grown at the apex of a $\mathrm{Si}_{3} \mathrm{~N}_{4}$ pyramid. To be noted both the shape and symmetry of the profile, the latter being due to the C-tip scanning the sample perpendicularly to its surface.

Carbon nano-tips offer two great advantages compared to standard tips: (i) they reduce (and in some cases render negligible) the artefacts connected with the tip shape (without recourse to complex deconvolution procedures which require the knowledge of the tip shape and size) in all those cases where the specimen surface contains steep slants and, (ii) the adhesion forces between the tip and the sample are reduced by a factor of about 10, as can be seen in Figure 9; this advantage, of paramount importance in the observation of soft organic and biological samples, results from the small apical size of the C-tips and possibly from the water/carbon interaction. It has been noted that the C-tips are brittle and tend to chip off in the presence of lateral forces (Fig. 10), whereas the standard tips are much more robust.

\subsection{Field Emission with C-Tip}

Tips fabricated with the method described in Section 3 have been grown at the apex of electron microscope tungsten filaments and tested for emission in a ultra-high vacuum system (pressure less than $5 \times 10^{-9}$ Torr). Field emission takes place, and currents from a few pA up to $0.2 \mu \mathrm{A}$ 
tip-sample approaching

tip-sample retracting
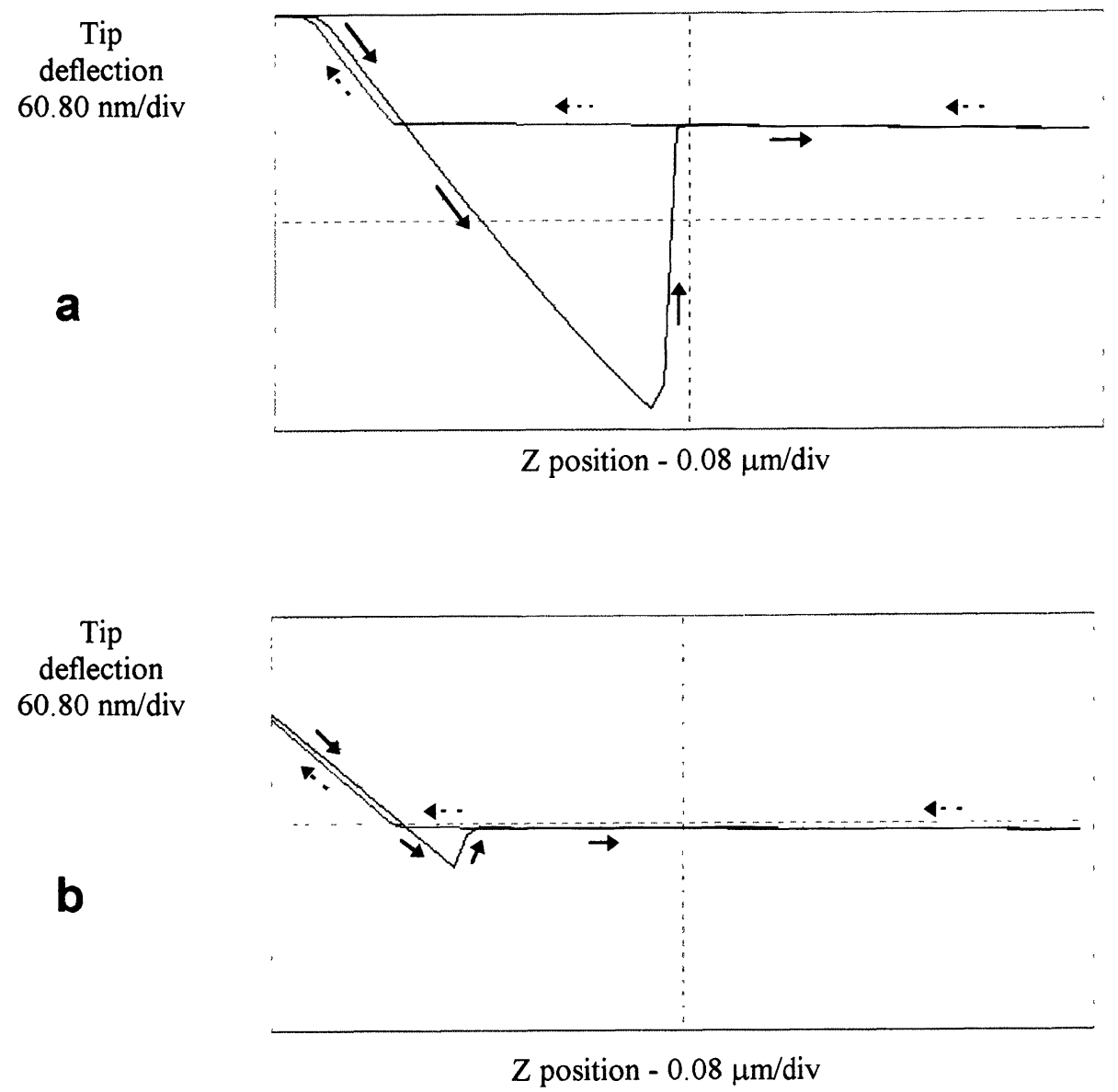

Fig. 9. - Force-distance curves obtained with the same cantilever, showing the different capillary forces acting on a calibration standard: (a) in the case of a conventional $\mathrm{Si}_{3} \mathrm{~N}_{4}$ pyramidal tip and (b) in the case of a carbon nano-tip grown on it.

have been measured on changing the extraction voltage. Figure 11 shows a typical result obtained. The change of slope during this run is attributed to a change in the emission area, possibly due to contamination or rearrangement of the tip atoms.

We are currently investigating the possibility of improving the emission stability.

\section{Conclusions}

An optimised method has been presented for growing amorphous carbon tips by carbon contamination inside a scanning electron microscope. The method is simple, effective, reliable, versatile, controllable and cheap. It is performed on a conductive surface, at an accelerating voltage of $30 \mathrm{kV}$; however, the use of higher voltages, if available, will be advantageous. The main features of the method are: use of a carbon stub (or a carbon block) as a source of 




Fig. 10. - SEM secondary electron image of latex spheres deposited on a gold-coated graphite surface after several observations in an AFM. To be noted the two broken tips lying near the large sphere. The marker represents $1 \mu \mathrm{m}$.

contaminant, and controlled focusing and astigmatism correction during the tip growth. The results can be immediately assessed.

Probes can be prepared with this technique in about $60 \mathrm{~s}$; they have a conical shape with the full angle as small as $10^{\circ}$ and the length of $1 \mu \mathrm{m}$. However, the height can be varied within a large range (from less than $1 \mu \mathrm{m}$ to several tens of $\mu \mathrm{m}$ ) by varying the contamination time. An example of application in the imaging and measurement of depth and size of blind holes has been given and comparison has been made to the case of using standard tips. It has also been shown that the capilary forces acting on a carbon nano-tip are smaller by one order of magnitude with respect to those acting on standard tips under the same conditions.

Nano-tips made by carbon contamination (radius less than $5 \mathrm{~nm}$ ) have been grown at the apex of ordinary electron microscope tungsten filaments, and field emission has been demonstrated to take place; its features are similar to those obtained with pointed tungsten filaments under the same geometrical conditions. However, current instabilities are a problem, which are believed to be related to the pressure in the field emission chamber, around $5 \times 10^{-9}$ Torr. Modifications to the chamber to improve the vacuum are in progress.

We envisage the exploitation of such arrangements in electron microscopes equipped with field emission guns. The use of standard pointed-filament (with their own sockets) at the top of which carbon nano-tips are grown under controlled conditions in a scanning electron microscope will allow their re-utilization in TEM/FEGs or in STEMs without the need to change neither the configuration of the gun electrodes, nor the related electronics, as might be required for re-sharpening blunt tungsten tips for field emission guns [12]. Carbon tips grown on an electrically conducting substrate may also be used in scanning tunneling microscopy. 


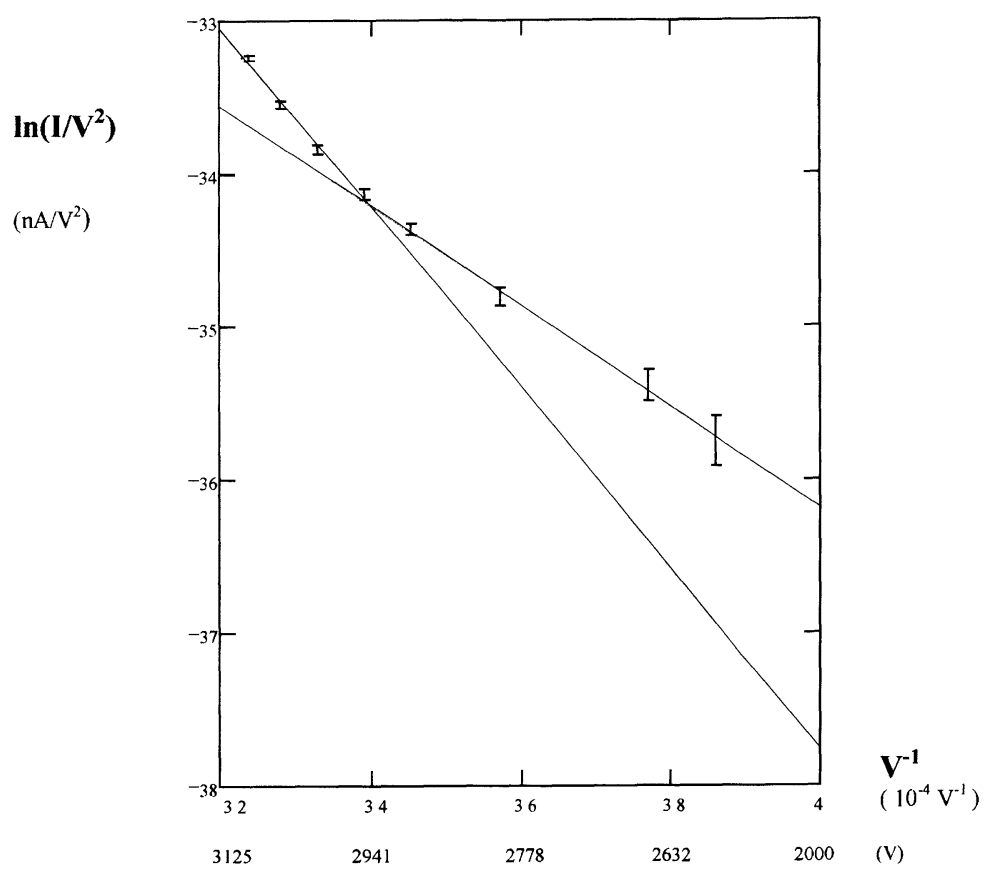

Fig. 11. - Experimental Fowler-Nordheim plot obtained from a carbon-contamination grown nanotip used as field emitter at a pressure of less than $5 \times 10^{-9}$ Torr. At $2600 \mathrm{~V}$ the emission current is $2.6 \mathrm{nA}$. The two trends are attributed to emission changes due to contaminants or geometrical changes.

\section{Acknowledgments}

We thank Dr A. Alessandrini for useful discussions and MURST for financial support.

\section{References}

[1] Keller D.J. and Chou C.-C., Surf. Sci. 268 (1992) 333.

[2] Fink H.W., Phys. Scr. 38 (1988) 260.

[3] Binh V.T. and Garcia N., Ultramicroscopy 42-44 (1992) 80.

[4] Baptist R., Proc. 7th Int. Vacuum Microelectronics Conf., Grenoble (Société Française du Vide, Paris, 1994).

[5] Rinzler A.S., Hafner J.H., Nikolaev P., Lou L., Kim S.G., Tomanek D., Nordlander P., Colbert D.T. and Smalley R.E., Science 269 (1995) 1150.

[6] De Heer W.A., Châtelain A. and Ugarte D., Science 270 (1995) 1179.

[7] Randi M.R., Monteverde F.T. and Valdrè U., J. Supercond. Sci. Technol. 7 (1994) 507.

[8] Hart K.R., Kassner T.F. and Maurin J.K., Phil. Mag. 21 (1970) 453.

[9] Hirsch P., Kässens M., Püttmann M. and Reimer L., Scanning 16 (1994) 101.

[10] Sentimenti A., Thesis, no. 2091, Università degli Studi, Bologna, 1995.

[11] Antognozzi M. and Valdrè U., Microsc. Microanal. Microstruct. 6 (1995) 513.

[12] Binh V.T., Garcia N. and Purcell S.T., Adv. Imag. Elec. Phys. 95 (1996) 63. 